\title{
ESTOQUE E DISPONIBILIDADE DE NITROGÊNIO NO SOLO EM EXPERIMENTO DE LONGA DURAÇÃO ${ }^{(1)}$
}

\author{
Mirla Andrade Weber ${ }^{(2)}$ \& João Mielniczuk ${ }^{(3)}$
}

\begin{abstract}
RESUMO
$\mathrm{O} \mathrm{N}$ é o nutriente requerido em maior quantidade pelas culturas agrícolas em geral e, normalmente, é o maior limitante de seu rendimento. Neste estudo, objetivou-se avaliar o efeito de sistemas de culturas no estoque de $\mathrm{N}$ total do solo e na disponibilidade deste nutriente. $O$ estudo foi desenvolvido em um experimento de longa duração (22 anos), em um Argissolo Vermelho distrófico típico localizado na Estação Experimental Agronômica da Universidade Federal do Rio Grande do Sul ( $30^{\circ} 50$ ' 52 " sul e $51^{\circ} 38$ ' 08 " oeste), em Eldorado do Sul, RS. Este experimento iniciou-se em 1983 e está sendo realizado desde sua implantação sob sistema plantio direto, com dez sistemas de culturas (solo descoberto, pousio/milho, aveia/milho, pangola, aveia + ervilhaca/milho com solo corrigido e revolvido até $70 \mathrm{~cm}$ em 1992, aveia + ervilhaca/milho, aveia + ervilhaca/milho + caupi, guandu + lablabe, lablabe + milho e guandu + milho) e duas doses de $\mathrm{N}\left(0 \mathrm{e} 180 \mathrm{~kg} \mathrm{ha}^{-1}\right)$ aplicadas no milho. Foram avaliados os estoques de $\mathrm{N}$ total do solo na camada de 0-20 cm em 2005 e estimadas as quantidades de $\mathrm{N}$ fixadas pelas leguminosas e perdidas do adubo nitrogenado mineral em 22 anos. Adicionalmente, foi avaliado o $\mathrm{N}$ acumulado pela aveia cultivada em sucessão ao milho em 2006. O estoque de $\mathbf{N}$ total, que era de

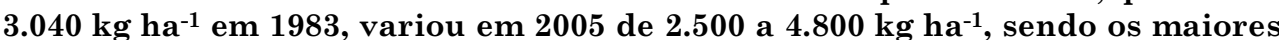
estoques encontrados nos sistemas com leguminosas e adubação nitrogenada. A quantidade estimada de $\mathrm{N}$ fixado pelas leguminosas variou de 800 a $1.980 \mathrm{~kg} \mathrm{ha}^{-1} \mathrm{e}$, na média, $48 \%$ do $\mathrm{N}$ mineral aplicado no milho $\left(3.180 \mathrm{~kg} \mathrm{ha}^{-1}\right)$ foi perdido do sistema solo-planta. Os maiores estoques de $\mathrm{N}$ total refletiram-se nas maiores quantidades de $\mathrm{N}$ acumulado pela aveia que variaram de 17 a $100 \mathrm{~kg} \mathrm{ha}^{-1}$.
\end{abstract}

Termos para indexação: sistemas de culturas, leguminosas, plantio direto, plantas de cobertura.

\footnotetext{
(1) Parte da Dissertação de Mestrado do primeiro autor. Recebido para publicação em julho de 2008 e aprovado em janeiro de 2009.

(2) Doutoranda do Programa de Pós-Graduação em Ciência do Solo da Universidade Federal do Rio Grande do Sul - UFRGS. Av. Bento Gonçalves 7712, Caixa Postal 776, CEP 91540-000 Porto Alegre (RS). E-mail: alrimbr@yahoo.com.br

(3) Professor do Departamento de Solos, UFRGS. Bolsista CNPq. E.mail: 00002273@ufrgs.br.
} 


\title{
SUMMARY: SOIL NITROGEN STOCKAND AVAILABILITY INA LONG-TERM EXPERIMENT
}

\begin{abstract}
Nitrogen is the nutrient required in the largest quantity by plants and is generally the most limiting nutrient for crop yield. The objective of this research was to evaluate the effect of cropping systems on Naccumulation and availability in the soil. A long-term experiment (22 years) was conducted on a Paleudult soil at an experimental station of the Federal University of Rio Grande do Sul (30 $50^{\circ} 52$ " $S$ and $51^{\circ} 38^{\prime} 08^{\prime}$ W), in Eldorado do Sul, Brazil, established in 1983. The experiment consisted of ten no-till cropping systems (bare soil, fallow/ maize, oat/maize, Digitaria, oat + vetch/maize limed and mobilized to a depth of $70 \mathrm{~cm}$ in 1992, oat + vetch/maize, oat+vetch/maize+cowpea, pigeon pea+lablab, lablab+maize, pigeon pea + maize,) and two $\mathrm{N}$ rates applied to maize as urea (0 and $\left.180 \mathrm{~kg} \mathrm{ha}^{-1}\right)$. Soil was sampled in 2005 from the $0-20 \mathrm{~cm}$ layer and $N$ stocks were determined. The amounts of biologically fixed $N$ by legumes and $N$ lost from fertilizer (urea) in 22 years were estimated. The $N$ uptake by oat grown in succession to maize in 2006 was also evaluated. The $N$ stock amounted to $3,040 \mathrm{~kg} \mathrm{ha}^{-1}$ in 1983 and ranged from 2,500 to $4,800 \mathrm{~kg} \mathrm{ha}^{-1}$ in 2005 . The legume-based cropping systems resulted in the highest $N$ stocks. Biological $N$ fixation ranged from 800 to

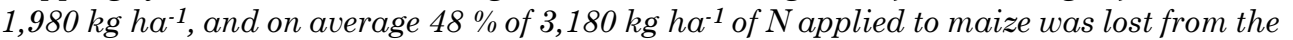
soil-plant system. The greatest $N$ stocks in soil under legume-based systems resulted in the greatest oat $\mathrm{N}$ uptake, which ranged from 17 to $100 \mathrm{~kg} \mathrm{~N} \mathrm{ha}^{-1}$.
\end{abstract}

Index terms: cropping systems, legumes, no-tillage, cover crops.

\section{INTRODUÇÃO}

A matéria orgânica do solo (MOS) constitui a principal fonte de $\mathrm{N}$ para as plantas. Entretanto, ela não consegue atender a alta demanda de $\mathrm{N}$ pelas culturas comerciais, principalmente em solos degradados, onde o baixo conteúdo de MOS torna a disponibilidade de $\mathrm{N}$ ainda menor. Por essa razão, a adição de fertilizantes nitrogenados minerais ou a utilização de leguminosas torna-se necessária para aumentar a disponibilidade de $\mathrm{N}$ em agroecossistemas (Peoples et al., 1995).

As leguminosas destacam-se como plantas de cobertura ou adubos verdes pela redução da erosão do solo, conservação da água no solo e reciclagem de nutrientes. Porém, o grande benefício do cultivo de leguminosas no aumento do rendimento das culturas tem sido atribuído ao aumento da disponibilidade de N às culturas cultivadas em sucessão (Fugita et al., 1992; Da Ros \& Aita, 1996; Rao \& Mathuva, 2000; Aita et al., 2001), permitindo a redução dos custos com fertilizantes nitrogenados minerais (Bohlool et al., 1992) e dos impactos ambientais gerados pela produção industrial desses fertilizantes, o que envolve alto consumo de combustíveis fósseis, com significativa emissão de gases para a atmosfera (Amado et al., 2001; Zanatta et al., 2007). A utilização de leguminosas em sistemas de culturas associada ao sistema plantio direto também contribui para o aumento dos estoques de $\mathrm{N}$ total do solo (Poudel et al., 2001; Diekow et al., 2005) como resultado da maior entrada de $\mathrm{N}$ no solo pela fixação biológica realizada pelas leguminosas somada à menor taxa de mineralização do N orgânico em sistema plantio dire- to (Bayer \& Mielniczuk, 1997; Lovato et al., 2004), podendo recuperar a capacidade de fornecimento de $\mathrm{N}$ pelo solo às culturas. Sainju et al. (2002) encontraram, após três anos, acúmulo de $430 \mathrm{~kg} \mathrm{ha}^{-1}$ até $20 \mathrm{~cm}$ de profundidade no estoque de $\mathrm{N}$ total do solo com a utilização de ervilhaca e tomate em sistema plantio direto. Já no mesmo sistema, porém sem leguminosas, houve queda de $250 \mathrm{~kg} \mathrm{ha}^{-1}$ de N.

Como observado em estudos anteriores, o acúmulo de $\mathrm{N}$ total no solo ocorre lentamente. Por isso, para a observação do impacto de práticas de manejo, como a utilização de sistemas de culturas, sobre a disponibilidade e acúmulo de $\mathrm{N}$ no solo são necessários experimentos de longa duração.

Diante do exposto, este estudo teve como objetivo avaliar o efeito do uso de diferentes sistemas de culturas de gramíneas e leguminosas sobre a disponibilidade e estoque de $\mathrm{N}$ no solo e o rendimento de grãos de milho em um experimento realizado por 22 anos, em um Argissolo Vermelho distrófico típico, em condições edafoclimáticas da região da Depressão Central do Rio Grande do Sul. Além disso, objetivouse estimar a quantidade de $\mathrm{N}$ exportada pela colheita de grãos, a fixada pelas leguminosas e a perdida do adubo mineral aplicado no milho nesse período.

\section{MATERIAL E MÉTODOS}

Este estudo foi desenvolvido em um experimento de longa duração instalado em 1983 num Argissolo Vermelho distrófico típico (Embrapa, 1999), que se 
encontrava degradado pelo cultivo de colza e girassol em preparo convencional (Medeiros, 1985), localizado na Estação Experimental Agronômica da Universidade Federal do Rio Grande do Sul ( $30^{\circ} 50$ ' 52 " sul e $51^{\circ} 38^{\prime} 08$ " oeste), no município de Eldorado do Sul, na região da Depressão Central do RS, a uma altitude média de $46 \mathrm{~m}$. O clima predominante é o subtropical úmido de verão quente do tipo fundamental Cfa, conforme a classificação de Köppen (Moreno, 1961). O delineamento experimental foi de blocos casualizados, com parcelas subdividas e três repetições. Nas parcelas principais, de 8 × $5 \mathrm{~m}$, foram cultivados dez sistemas de culturas em semeadura direta (Quadro 1) e, nas subparcelas, de $4 \times 5 \mathrm{~m}$, foram aplicadas duas doses de $\mathrm{N}$ na forma de uréia na cultura do milho. As doses de N constituíram-se de 0 e $120 \mathrm{~kg} \mathrm{ha}^{-1}$ de 1983 a 1993 e, a partir de 1994, a dose de 120 foi elevada para $180 \mathrm{~kg} \mathrm{ha}^{-1}$, totalizando $3.180 \mathrm{~kg} \mathrm{ha}^{-1}$ de $\mathrm{N}$ aplicados na cultura do milho nos sistemas $\mathrm{P} / \mathrm{M}, \mathrm{A} / \mathrm{M}, \mathrm{A}+\mathrm{V} / \mathrm{M}$, $\mathrm{A}+\mathrm{V} / \mathrm{M}+\mathrm{C}, \mathrm{G}+\mathrm{M}$ e LL+M (Quadro 1). Nos sistemas G+LL, Pan e Desc foi cultivado milho nas safras 1988/ $1989,1993 / 1994,1995 / 1996$ e $1996 / 1997$, os quais receberam $\mathrm{N}$ mineral apenas nesses anos. Já no sistema A+V/M rev, o milho foi cultivado a partir da safra 1988/1989, recebendo aplicação de N a partir desta data. Durante o desenvolvimento da cultura do milho, foram realizadas irrigações.

Em 2005 foram coletadas amostras de solo nas profundidades de $0-10$ e $10-20 \mathrm{~cm}$ em todas as parcelas. O solo amostrado foi submetido à análise de $\mathrm{N}$ total, segundo Tedesco et al. (1995). Os estoques de $\mathrm{N}$ total foram calculados utilizando o método da massa equivalente (Bayer, 2003), tendo como referência as densidades do solo obtidas em campo nativo adjacente ao experimento, 1,54 e 1,63 $\mathrm{Mg} \mathrm{m}^{-3}$ para as camadas de $0-10 \mathrm{~cm}$ e $10-20 \mathrm{~cm}$, respectivamente (Lovato, 2001). Este método foi escolhido para se evitar a superestimativa do acúmulo de $\mathrm{N}$ total nos tratamentos com maior densidade. Neste método de cálculo, a massa de solo do campo nativo, obtida por meio de sua densidade de solo nas camadas de $0-10$ e de $10-20 \mathrm{~cm}$ de profundidade, é considerada como referência, sendo denominada massa equivalente. A partir da massa equivalente e das densidades de solo das camadas de cada tratamento não referência, obtidas neste experimento por Bayer (1996), calcularam-se as espessuras das camadas de solo requeridas para atingir uma massa equivalente à massa de solo do sistema de referência, objetivando igualar as massas de solo de cada tratamento. Detalhes do procedimento de cálculo desse método encontram-se em Ellert \& Bettany (1995). O estoque inicial do solo, $3.040 \mathrm{~kg} \mathrm{ha}^{-1}$ de N na camada $0-20 \mathrm{~cm}$ (Lovato, 2001), foi utilizado para calcular a variação no estoque em 22 anos.

A partir dos dados de rendimento de grãos de milho das safras de 1984/1985 a 2004/2005 (Weber, 2008), exceto nos sistemas G+LL, Pan e Desc, foram estimadas as quantidades de $\mathrm{N}$ exportadas pela colheita dos grãos de milho em todo o período experimental por meio de um valor médio obtido por dois métodos. $\mathrm{O}$ primeiro método estimou o $\mathrm{N}$ exportado pela colheita de grãos por meio da equação apresentada por Lovato (2001):

$$
\hat{y}=-10,08+13,76 x
$$

em que ŷ é a quantidade de $\mathrm{N}$ absorvido pelos grãos de milho até a maturação fisiológica, que foi exportada pela colheita, em kg ha-1, e x é o rendimento de grãos de milho, em Mg ha ${ }^{-1}$.

Já no segundo método, utilizaram-se as concentrações de $\mathrm{N}$ no grão do milho de cada tratamento, no momento da colheita da safra 2005/ 2006 (Weber, 2008), para estimar a quantidade de $\mathrm{N}$ exportado pelas safras anteriores.

Para se estimar o $\mathrm{N}$ fixado pelas leguminosas e o $\mathrm{N}$ perdido do adubo mineral em todo o período experimental, primeiramente estimou-se a quantidade de $\mathrm{N}$ exportado absorvida da camada abaixo de $20 \mathrm{~cm}$,

\section{Quadro 1. Composição dos sistemas de culturas estudados}

\begin{tabular}{lll}
\hline Sistema & \multicolumn{1}{c}{ Inverno } & \multicolumn{1}{c}{ Verão } \\
\hline $\mathrm{A} / \mathrm{M}$ & Aveia (Avena strigosa) & Milho (Zea mays) \\
$\mathrm{A}+\mathrm{V} / \mathrm{M}$ & Aveia + Ervilhaca (Vicia sativa) & Milho \\
$\mathrm{A}+\mathrm{V} / \mathrm{M} \mathrm{rev}{ }^{(1)}$ & Aveia + Ervilhaca & Milho \\
$\mathrm{A}+\mathrm{V} / \mathrm{M}+\mathrm{C}$ & Aveia + Ervilhaca & Milho + Caupi (Vignea unguiculata) \\
Desc & Solo descoberto & Solo descoberto \\
$\mathrm{G}+\mathrm{LL}$ & Guandu (Cajanus cajan) & Guandu + Lablabe (Dolichos lablab) \\
$\mathrm{G}+\mathrm{M}$ & Guandu & Guandu + Milho \\
$\mathrm{LL}+\mathrm{M}$ & Pousio & Lablabe + Milho \\
$\mathrm{P} / \mathrm{M}$ & Pousio & Milho \\
$\mathrm{Pan}$ & Pangola (Digitaria) & Pangola \\
\hline
\end{tabular}

\footnotetext{
${ }^{(1)}$ Solo corrigido e revolvido até $70 \mathrm{~cm}$ em 1992.
} 
nos tratamentos $\mathrm{A} / \mathrm{M}$ e $\mathrm{P} / \mathrm{M}$ sem adubação nitrogenada mineral, pois nesses tratamentos a quantidade de $\mathrm{N}$ exportada pelos grãos foi maior do que o decréscimo do estoque de $\mathrm{N}$ total na camada de $0-20 \mathrm{~cm} \mathrm{em}$ 22 anos. Para este cálculo, utilizou-se a seguinte equação:

$$
\mathrm{N}_{+20 \mathrm{~cm}}=\mathrm{N}_{\text {exp }}-\Delta \mathrm{NT}
$$

em que $\mathrm{N}_{+20 \mathrm{~cm}}$ é o $\mathrm{N}$ exportado pelos grãos que foi absorvido abaixo de $20 \mathrm{~cm}$, em kg ha-1 $\mathrm{N}_{\exp }$ é o $\mathrm{N}$ total exportado pelos grãos, em $\mathrm{kg} \mathrm{ha}^{-1}$; e $\Delta \mathrm{NT}$ é a variação no estoque de $\mathrm{N}$ total, em $\mathrm{kg} \mathrm{ha}^{-1}$, calculada da seguinte maneira:

$$
\Delta \mathrm{NT}=\mathrm{NT}_{2005}-\mathrm{NT}_{1983}
$$

em que $\mathrm{NT}_{2005}$ é o estoque de $\mathrm{N}$ total até $20 \mathrm{~cm}$ em 2005, em kg ha-1, e $\mathrm{NT}_{1983}$ é o estoque de $\mathrm{N}$ total até $20 \mathrm{~cm}$ em 1983, em kg ha-1.

Posteriormente, foram estimadas as quantidades de $\mathrm{N}$ fixado pelas leguminosas nos tratamentos $\mathrm{A}+\mathrm{V} /$ $\mathrm{M}$ rev, A+V/M, A+V/M+C, G+M e LL+M sem adubação nitrogenada mineral e as perdidas do adubo mineral aplicado no milho nos tratamentos A/M e P/M. Para isso, foram realizados os seguintes cálculos:

$$
\begin{gathered}
\mathrm{N}_{\text {fix }}=\Delta \mathrm{NT}+\left(\mathrm{N}_{\text {exp }}-\mathrm{N}_{+20 \mathrm{~cm}}\right) \\
\mathrm{N}_{\text {perd }}=\mathrm{N}_{\text {apl }}-\Delta \mathrm{NT}-\left(\mathrm{N}_{\text {exp }}-\mathrm{N}_{+20 \mathrm{~cm}}\right)
\end{gathered}
$$

em que $\mathrm{N}_{\text {fix }}$ é a quantidade de $\mathrm{N}$ fixada pelas leguminosas em kg ha-1; $\mathrm{N}_{\text {perd }}$ é a quantidade de $\mathrm{N}$ que foi perdida do adubo nitrogenado aplicado no milho, em kg ha-1; e $\mathrm{N}_{\text {apl }}$ é a quantidade de $\mathrm{N}$ que foi aplicada na cultura do milho $\left(3.180 \mathrm{~kg} \mathrm{ha}^{-1}\right)$. Essas quantidades são referentes a todo o período experimental.

$\mathrm{O} \mathrm{N}+20 \mathrm{~cm}$ obtido nos tratamentos $\mathrm{P} / \mathrm{M}$ e $\mathrm{A} / \mathrm{M}$ sem adição de adubação nitrogenada mineral foi de $200 \mathrm{~kg} \mathrm{ha}^{-1}$, quantidade referente a 21 colheitas de grãos ocorridas nesses dois sistemas. Este valor de $\mathrm{N}_{+20 \mathrm{~cm}}$, $200 \mathrm{~kg} \mathrm{ha}^{-1}$, foi utilizado para se estimar o $\mathrm{N}$ fixado pelos sistemas $\mathrm{A}+\mathrm{V} / \mathrm{M}$ e $\mathrm{A}+\mathrm{V} / \mathrm{M}+\mathrm{C}$, conforme a equação 4. Entretanto, nos sistemas A+V/M rev, $\mathrm{G}+\mathrm{M}$ e LL+M, por terem ocorrido 15, 16 e 19 colheitas, respectivamente, e não 21 como nos sistemas $\mathrm{P} / \mathrm{M}$ e A/M, foram utilizados os valores de 140, 150 e $180 \mathrm{~kg} \mathrm{ha}^{-1}$, respectivamente, proporcionais ao número de colheitas, como a quantidade de N exportado pelos grãos proveniente da camada abaixo dos $20 \mathrm{~cm}$ de profundidade, para se estimar a quantidade de $\mathrm{N}$ fixada pelas leguminosas desses três sistemas. $\mathrm{O}$ menor número de safras dos tratamentos $\mathrm{G}+\mathrm{M}$ e LL+M deve-se a problemas de desenvolvimento da cultura do milho em alguns anos que acarretaram na ausência de colheita de grãos. Para estimar as perdas de $\mathrm{N}$ do fertilizante aplicado no milho também foi utilizado o valor de $200 \mathrm{~kg} \mathrm{ha}^{-1}$ como a quantidade de $\mathrm{N}$ exportada proveniente da camada abaixo dos $20 \mathrm{~cm}$ de profundidade (Equação 5).
O efeito residual dos diferentes sistemas de culturas na disponibilidade de $\mathrm{N}$ foi avaliado por meio da quantidade de $\mathrm{N}$ que foi reciclada pela parte aérea da aveia-preta cultivada em sucessão ao milho da safra 2005/2006 nas parcelas de todos os tratamentos sem histórico de aplicação de adubo nitrogenado mineral na cultura do milho. A aveia foi coletada numa área de $0,5 \mathrm{~m}^{2}$ em setembro de 2006, quando se encontrava no final do florescimento. A concentração de $\mathrm{N}$ no tecido da aveia foi determinada pelo método de Tedesco et al. (1995) e a absorção de $\mathrm{N}$ pela biomassa da parte aérea da aveia calculada em $\mathrm{kg} \mathrm{ha}^{-1}$.

$\mathrm{O}$ efeito dos diferentes sistemas de culturas e doses de $\mathrm{N}$ sobre os estoques de $\mathrm{N}$ total no solo tiveram sua significância avaliada pela análise de variância e a diferença entre médias de tratamentos foi obtida pelo teste de Tukey a $5 \%$, para os sistemas de culturas, e a $10 \%$ para as doses de $\mathrm{N}$ dentro de cada sistema de cultura. Para a equação de regressão, o coeficiente de determinação teve sua significância testada pelo teste F a 1 \%. Em relação ao rendimento de grãos de milho, média de todas as safras, os tratamentos tiveram sua significância avaliada pela análise de variância e a diferenças entre médias pelo teste de Tukey a $5 \%$. Para esta análise, os anos foram considerados como repetições, pois, como se trata de um experimento de longa duração (22 anos), em alguns anos não se dispõe de repetições, mas apenas médias de cada tratamento. Para a análise estatística dos dados foi utilizado o programa computacional SANEST (Zonta \& Machado, 1984).

\section{RESULTADOS E DISCUSSÃO}

\section{N exportado pela colheita de milho e rendi- mento de grãos}

Diferenças significativas foram observadas entre os sistemas de culturas e as doses de N no rendimento médio de grãos de milho, sendo observada interação entre os dois fatores. Na ausência de adubação nitrogenada mineral, os sistemas com leguminosas apresentaram maiores rendimentos de grãos na média do período 1984-2005 (Figura 1a). Com a aplicação de adubação nitrogenada mineral na cultura do milho, houve aumento no rendimento de grão. Entretanto, nesta situação não houve diferença estatística entre os sistemas de culturas no rendimento de grãos. O sistema $\mathrm{A}+\mathrm{V} / \mathrm{M}$ rev, em ambas as doses de $\mathrm{N}$, foi o sistema que apresentou tendência clara de maior rendimento de grãos. Nesse sistema, o revolvimento do solo até $70 \mathrm{~cm}$ de profundidade em 1992, somado à calagem e à adição de $\mathrm{P}$, proporcionou melhorias nas condições do solo no ambiente radicular, determinando que a cultura do milho expressasse melhor seu potencial produtivo (Fernandes, 1998). 
A colheita dos grãos de milho determinou que parte do $\mathrm{N}$ que entrou no sistema solo-planta, por fixação biológica de $\mathrm{N}_{2}$ (FBN) ou adubação mineral, fosse exportada (Figura 1b). À semelhança dos rendimentos de grãos, as maiores exportações aconteceram nos sistemas com maior disponibilidade de $\mathrm{N}$, com leguminosas e, ou, adubação nitrogenada mineral. Nos sistemas A/M e P/M sem adubação nitrogenada, houve exportação de mais de $400 \mathrm{~kg} \mathrm{ha}^{-1}$ de $\mathrm{N}$ em todo o período experimental, $\mathrm{N}$ este proveniente
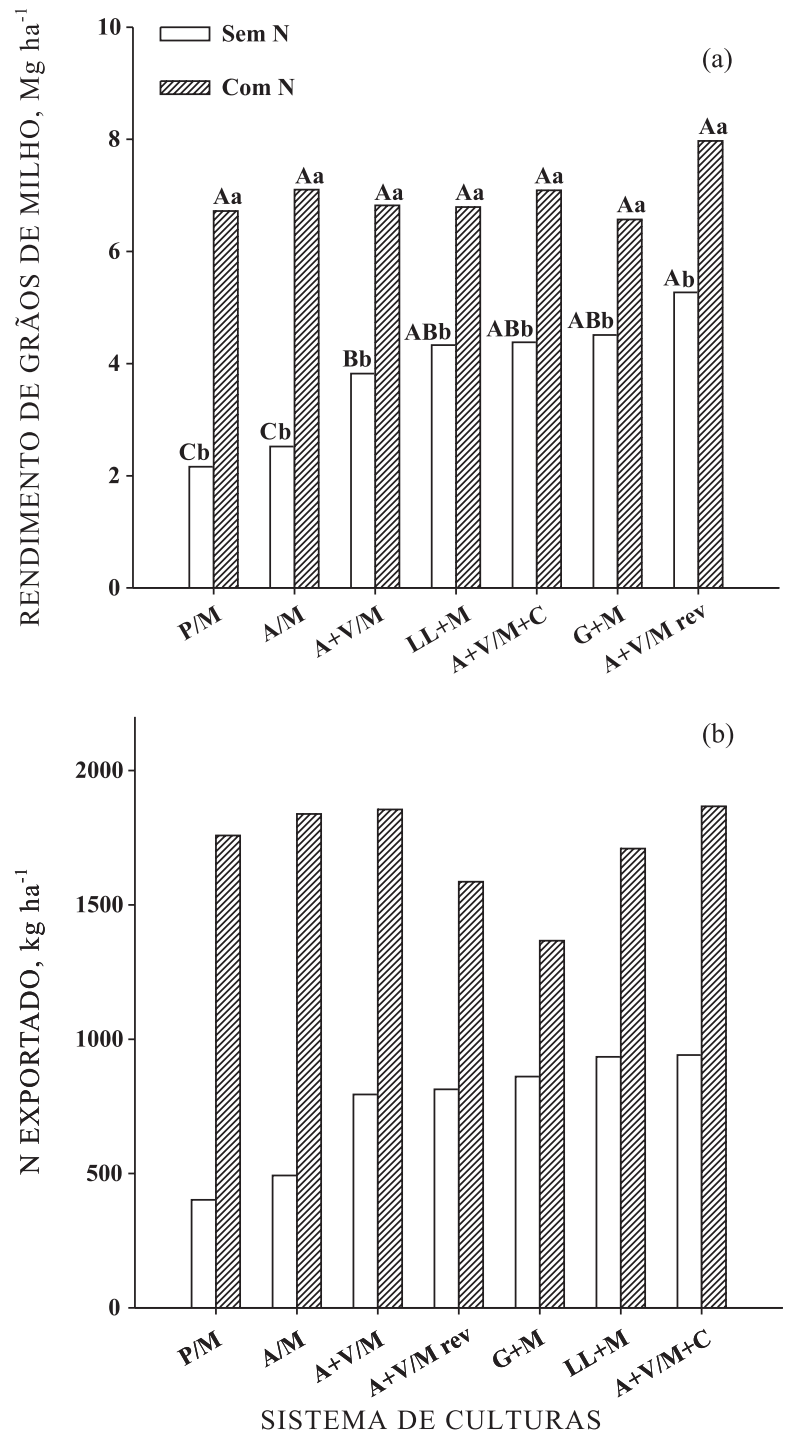

Figura 1. Rendimento de grãos de milho, $13 \%$ de umidade (a), média das safras de 1984/1985 a 2004/2005, e quantidade de $\mathrm{N}$ exportado pela colheita de grãos de milho nesse período (b) em diferentes sistemas de culturas e em duas doses de N. Letras maiúsculas comparam sistemas de culturas dentro de cada dose de $\mathrm{N}$ e letras minúsculas comparam doses de $\mathrm{N}$ dentro de cada sistema de cultura, por meio do teste de Tukey a $5 \%$. A: aveia, C: caupi, G: guandu, LL: lablabe, M: milho, P: pousio, rev: revolvido e V: ervilhaca. exclusivamente do estoque de $\mathrm{N}$ total do solo. Já nos sistemas A/M, P/M, A+V/M e A+V/M+C com adubação nitrogenada, a exportação chegou a ultrapassar $1.800 \mathrm{~kg} \mathrm{ha}^{-1} \mathrm{de} \mathrm{N}$, proveniente do solo e do adubo nos dois primeiros e nos demais das três fontes: solo, adubo mineral e FBN.

\section{Estoques de $\mathbf{N}$ total}

Os sistemas de culturas e as doses de $\mathrm{N}$ aplicadas determinaram diferenças significativas nos estoques de $\mathrm{N}$ total em 2005, não sendo observada interação significativa entre esses dois fatores (Figura 2). Houve acúmulo de $\mathrm{N}$ total nos sistemas com leguminosas em relação ao início do experimento, resultado da adição de N por esses sistemas via FBN realizada pelas leguminosas.

A adição de $\mathrm{N}$ pelas leguminosas nestes sistemas é observada na maior quantidade de $\mathrm{N}$ acumulado pela biomassa das culturas de inverno e verão desses sistemas em relação àqueles constituídos apenas por gramíneas. Burle et al. (1997) encontraram quantidades de $\mathrm{N}$ acumulado pelas culturas de inverno e verão de 53, 143 e $234 \mathrm{~kg} \mathrm{ha}^{-1}$ ano $^{-1}$, média dos 10 primeiros anos do experimento deste estudo, respectivamente, para os sistemas constituídos apenas por gramíneas, com leguminosas de inverno e com leguminosas de verão, todos sem adubação nitrogenada mineral. A diferença entre essas quantidades foi atribuída à FBN realizada pelas leguminosas, observando-se maior destaque para as

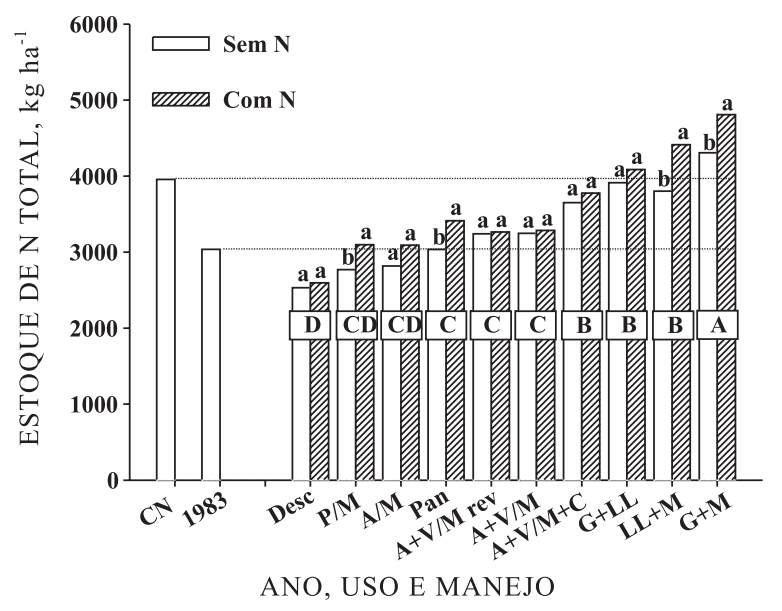

Figura 2. Estoques de $\mathrm{N}$ total na camada 0-20 cm do campo nativo (CN), no início do experimento (1983) e dos sistemas de culturas sob plantio direto com duas doses de nitrogênio mineral em 2005. Letras maiúsculas comparam sistemas de culturas (média das duas doses de $\mathrm{N}$ ) e letras minúsculas comparam doses de $\mathbf{N}$ dentro de cada sistema de cultura, por meio do teste de Tukey a $5 \%$ e $10 \%$, respectivamente. A: aveia, C: caupi, Desc: descoberto, G: guandu, LL: lablabe, M: milho, P: pousio, Pan: pangola, rev: revolvido e V: ervilhaca. 
leguminosas de ciclo estival. A adição de N pela FBN nestes sistemas também pode ser observada por meio da maior produção de biomassa vegetal desses sistemas, como observado por Vieira (2007), que encontrou adições de $\mathrm{C}$ pela biomassa vegetal dos sistemas com leguminosas de $6,56 \mathrm{Mg} \mathrm{ha}^{-1} \mathrm{ano}^{-1}$ contra $3,60 \mathrm{Mg} \mathrm{ha}^{-1}$ ano $^{-1}$ dos sistemas constituídos apenas por gramíneas, na média dos 19 anos deste experimento. Mais adições de $\mathrm{N}$ pelas culturas resultaram em mais acúmulo de $\mathrm{N}$ total no solo em sistema plantio direto $\left(R^{2}=0,97\right)$, em estudo desenvolvido por Lovato et al. (2004), no qual observouse que $50 \%$ do $\mathrm{N}$ adicionado pelas culturas foi retido como $\mathrm{N}$ total no solo.

Dentre as leguminosas, as estivais apresentaram destaque no acúmulo de $\mathrm{N}$ total no solo. Os sistemas sem adubação nitrogenada LL+M, G+LL e G+M acumularam no solo, em relação ao início do experimento, 770,870 e $1.270 \mathrm{~kg} \mathrm{ha}^{-1}$ de $\mathrm{N}$, o que equivale a uma taxa anual de acúmulo de 35,40 e $58 \mathrm{~kg} \mathrm{ha}^{-1} \mathrm{ano}^{-1} \mathrm{de} \mathrm{N}$, respectivamente. Esses estoques estão próximos ou já ultrapassaram aqueles do campo nativo. Já os sistemas com leguminosas de inverno apresentaram, em média, uma taxa de acúmulo de $9 \mathrm{~kg} \mathrm{ha}^{-1} \mathrm{ano}^{-1}$ de N.

O maior efeito das leguminosas de verão sobre os estoques de $\mathrm{N}$ total, comparativamente às de inverno, também foi observado por Amado et al. (2001). Os autores encontraram taxas de acúmulo de $160 \mathrm{~kg} \mathrm{ha}^{-1}$ $\mathrm{ano}^{-1}$ de N no sistema mucuna + milho em relação ao sistema pousio/milho, após oito anos de uso em Santa Maria, RS. Já os sistemas aveia+ervilhaca/milho e tremoço (azevém+ervilhaca)/milho apresentaram uma taxa de 81 e $75 \mathrm{~kg} \mathrm{ha}^{-1}$ ano $^{-1}$ de $\mathrm{N}$, respectivamente. Esses resultados foram atribuídos à maior adição anual de $\mathrm{N}$ ao solo pela parte aérea das culturas do sistema mucuna+milho $\left(217 \mathrm{~kg} \mathrm{ha}^{-1}\right)$, comparativamente aos sistemas com leguminosas de inverno anteriormente citados (141 e $117 \mathrm{~kg} \mathrm{ha}^{-1}$, respectivamente). Bayer et al. (2003) encontraram taxas de acúmulo de $240 \mathrm{~kg} \mathrm{ha}^{-1}$ ano $^{-1}$ de N, em cinco anos de utilização nos sistemas milho + mucuna e milho + feijão de porco em relação ao início do experimento conduzido em preparo reduzido, em Chapecó, SC. Na Austrália, em pastagens com trevo subterrâneo, foram encontradas taxas de acúmulo variando de 25 a $100 \mathrm{~kg} \mathrm{ha}^{-1} \mathrm{ano}^{-1}$ de N (Peoples et al., 1995).

Diante desses resultados, torna-se evidente o papel das leguminosas em determinar balanços positivos de N no sistema solo-planta. Entretanto, nem todos os sistemas que utilizam leguminosas determinam balanços positivos de N. No estudo de Alves et al. (2005), em que a leguminosa utilizada foi a soja, o balanço de $\mathrm{N}$ foi próximo da neutralidade devido a alta exportação de N pelos grãos desta cultura. Fugita et al. (1992) relataram que o sistema de cultura milho/ caupi determinou balanço negativo de $14 \mathrm{~kg} \mathrm{ha}^{-1} \mathrm{de}$ $\mathrm{N}$, proveniente da alta demanda de $\mathrm{N}$ pelo milho e exportação de $\mathrm{N}$ pela colheita de grãos desta cultura, enquanto o sistema constituído somente por caupi apresentou um balanço positivo de $36 \mathrm{~kg} \mathrm{ha}^{-1}$ de N.

A adubação nitrogenada promoveu acúmulo de $\mathrm{N}$ total no solo em relação à sua ausência, porém este aumento (13\%) foi significativo apenas nos sistemas P/M, LL+M, Pan e G+M (Figura 2). Lovato et al. (2004) também observaram aumento de $15 \%$, nos estoques de $\mathrm{N}$ total com esta prática. Nos sistemas $\mathrm{P} / \mathrm{M}$ e A/M (Figura 2) sem aplicação de adubo nitrogenado, houve um decréscimo médio, em relação a 1983, de $250 \mathrm{~kg} \mathrm{ha}^{-1}$ de N, proveniente da exportação de N pela colheita do grão. Porém, com a adição de $\mathrm{N}$, os sistemas acumularam $60 \mathrm{~kg} \mathrm{ha}^{-1}$ de $\mathrm{N}$, o que promoveu a manutenção do estoque inicial de $\mathrm{N}$ total. Este fato evidencia a importância da adubação nitrogenada em sistemas baseados exclusivamente em gramíneas a fim de evitar o esgotamento desse nutriente no solo.

No sistema Desc, a queda no estoque de N total, de $470 \mathrm{~kg}$ ha $^{-1}$, não se deve somente à exportação pela colheita, pois esta ocorreu apenas em quatro anos, mas, em parte, ao processo de erosão do solo, pois este sistema foi mantido descoberto em grande parte do período, e, principalmente, à lixiviação. Como neste sistema não houve plantas em desenvolvimento para acumular $\mathrm{N}$ em sua biomassa, o $\mathrm{N}$ mineralizado da MOS foi levado para camadas mais profundas pelo movimento de percolação da água.

\section{$\mathrm{N}$ fixado pelas leguminosas}

A quantidade estimada de $\mathrm{N}$ fixado pelas leguminosas em 22 anos variou de 800 a $1.980 \mathrm{~kg} \mathrm{ha}^{-1}$ (Quadro 2), com as maiores quantidades encontradas nos sistemas com leguminosas estivais. Estas quantidades demonstram o importante papel das leguminosas, principalmente as estivais, no aporte de N em sistemas agrícolas. Segundo Aita (1997), as leguminosas de verão na produção de matéria seca e quantidade de $\mathrm{N}$ acumulado são superiores às leguminosas de inverno, devido a sua rusticidade, que permite produzir expressivas quantidades de biomassa mesmo em condições de acidez e baixa fertilidade natural.

As quantidades estimadas correspondem a uma fixação de 36 a $90 \mathrm{~kg} \mathrm{ha}^{-1}$ ano $^{-1}$ de N, menores que as quantidades encontradas na literatura para as leguminosas deste estudo (Calegari et al., 1993; Peoples et al., 1995; Moreira \& Siqueira, 2006). Estas diferenças devem-se, em parte, às diferenças das condições edafoclimáticas dos ambientes estudados. Segundo Ledgard \& Steele (1992) e Moreira \& Siqueira (2006), a quantidade de $\mathrm{N}$ fixado pelas leguminosas depende das espécies, das condições climáticas e de solo. A temperatura e a umidade são os fatores climáticos mais importantes, enquanto o $\mathrm{pH}$, elementos tóxicos, teor de nutrientes, quantidade de $\mathrm{N}$ mineral e salinidade são os fatores edáficos que mais alteram a FBN. Entretanto, neste estudo não houve fatores químicos limitantes ao crescimento e desenvolvimento vegetal (Vieira, 2007). 
Quadro 2. Nitrogênio aparente fixado pelas leguminosas e nitrogênio perdido do fertilizante mineral aplicado na cultura do milho, em 22 anos, em diferentes sistemas de culturas

\begin{tabular}{|c|c|c|c|}
\hline Sistema de cultura & $\mathrm{N}$ fixado pelas leguminosas ${ }^{(1)}$ & $\mathrm{N}$ aplicado via fertilizante & $\mathrm{N}$ perdido do fertilizante ${ }^{(2)}$ \\
\hline & & $\mathrm{kg} \mathrm{ha}^{-1}$ & - \\
\hline $\mathrm{A}+\mathrm{V} / \mathrm{M}^{(3)}$ & 800 & 0 & - \\
\hline $\mathrm{A}+\mathrm{V} / \mathrm{M}$ rev & 870 & 0 & - \\
\hline $\mathrm{A}+\mathrm{V} / \mathrm{M}+\mathrm{C}$ & 1.350 & 0 & - \\
\hline $\mathrm{LL}+\mathrm{M}$ & 1.520 & 0 & - \\
\hline $\mathrm{G}+\mathrm{M}$ & 1.980 & 0 & - \\
\hline $\mathrm{P} / \mathrm{M}$ & - & 3.180 & 1.560 \\
\hline $\mathrm{A} / \mathrm{M}$ & - & 3.180 & 1.490 \\
\hline
\end{tabular}

${ }^{(1)} \mathrm{N}_{\text {fix }}=\Delta \mathrm{NT}+\left(\mathrm{N}_{\text {exp }}-\mathrm{N}_{+20 \mathrm{~cm}}\right) \cdot{ }^{(2)} \mathrm{N}_{\mathrm{perd}}=\mathrm{N}_{\mathrm{apl}}-\Delta \mathrm{NT}-\left(\mathrm{N}_{\mathrm{exp}}-\mathrm{N}_{+20 \mathrm{~cm}}\right) \cdot{ }^{(3)}$ A: aveia, C: caupi, G: guandu, LL: lablabe, M: milho, P: pousio, rev: revolvido e V: ervilhaca.

Este método, entretanto, subestimou a quantidade de $\mathrm{N}$ fixado, pois ele não considerou a quantidade de $\mathrm{N}$ perdida por meio dos vários processos de perdas a que o $\mathrm{N}$ está sujeito e o $\mathrm{N}$ fixado que foi acumulado na MOS abaixo dos $20 \mathrm{~cm}$ de profundidade. Em estudo realizado neste mesmo experimento, Diekow et al. (2005) investigaram o acúmulo de $\mathrm{N}$ total pelos diferentes sistemas de culturas até a profundidade de 107,5 cm. Em relação ao sistema A/M com adubação mineral, considerado o sistema que reproduziu a condição original do experimento, o sistema LL+M sem adubação acumulou $100 \mathrm{~kg} \mathrm{ha}^{-1}$ de N, abaixo dos $20 \mathrm{~cm}$ de profundidade. Porém, o sistema G+M sem adubação acumulou $1.210 \mathrm{~kg} \mathrm{ha}^{-1}$ de $\mathrm{N}$ em subsuperfície, o que correspondeu a $42 \%$ do total acumulado na camada de $0-107,5 \mathrm{~cm}$.

\section{$\mathrm{N}$ perdido do adubo mineral}

Dos $3.180 \mathrm{~kg} \mathrm{ha}^{-1}$ de $\mathrm{N}$ aplicados na cultura do milho nos sistemas $\mathrm{P} / \mathrm{M}$ e A/M, estimou-se que 1.560 e $1.490 \mathrm{~kg} \mathrm{ha}^{-1}$ foram perdidos, respectivamente (Quadro 2). Esses valores revelam que o sistema soloplanta recuperou, em média, $52 \%$ do $\mathrm{N}$ aplicado via fertilizante mineral. A planta de milho recuperou $49,5 \%$ por meio dos grãos, enquanto o solo recuperou apenas $2,5 \%$ do $\mathrm{N}$ do fertilizante mineral.

Os valores estimados correspondem aos valores mundiais de recuperação pela planta do $\mathrm{N}$ aplicado, que estão em torno de 50-60 \% (Cantarella, 2007). Entretanto, em estudo conduzido por Alves et al. (2005) no Mato Grosso do Sul, em um cultivo de milho, o sistema solo-planta recuperou 70,5\% dos $70 \mathrm{~kg} \mathrm{ha}^{-1}$ do $\mathrm{N}$ aplicado na forma de sulfato de amônio, sendo que $24,3 \%$ do $\mathrm{N}$ adicionado em cobertura encontravase na camada 0-20 cm após a colheita de milho. Gava et al. (2006) encontraram valores semelhantes: $45 \%$ e $30 \%$ dos $100 \mathrm{~kg} \mathrm{ha}^{-1}$ de $\mathrm{N}$ aplicados no milho na forma de uréia foram recuperados pela planta e pelo solo, respectivamente, até $50 \mathrm{~cm}$ de profundidade. A menor recuperação do $\mathrm{N}$ aplicado neste estudo, em relação aos de Alves et al. (2005) e Gava et al. (2006), deve-se à maior dose de $\mathrm{N}$ utilizada. Segundo Cantarella (2007), menores doses de $\mathrm{N}$ determinam maiores recuperações de $\mathrm{N}$.

Os menores valores encontrados neste estudo poderiam estar relacionados à não-contabilização do $\mathrm{N}$ aplicado, que pode ter sido imobilizado pelo solo e acumulado na MOS, abaixo de $20 \mathrm{~cm}$. Segundo Alves et al. (2005), as raízes abaixo desse limite podem recuperar parte do $\mathrm{N}$, não significando propriamente perdas. Entretanto, no estudo de Diekow et al. (2005), não houve diferença entre as doses 0 e $180 \mathrm{~kg} \mathrm{ha}^{-1}$ de $\mathrm{N}$ no acúmulo de $\mathrm{N}$ total abaixo de $20 \mathrm{~cm}$ no sistema $\mathrm{A} / \mathrm{M}$, o que indica que parte do $\mathrm{N}$ aplicado pelo fertilizante mineral não foi acumulado abaixo desta profundidade. Dessa forma, $48 \%$ do $\mathrm{N}$ aplicado no milho saiu do sistema solo-planta por meio das diversas formas de perdas a que o $\mathrm{N}$ está sujeito em sistemas agrícolas. Entretanto, como se trata de um experimento de longa duração, os processos de perdas podem ter atuado de forma diferente ao longo dos anos.

O método utilizado neste estudo, apesar de não obter resultados exatos, forneceu a magnitude da quantidade de $\mathrm{N}$ fixada nos diferentes sistemas de culturas e da quantidade de $\mathrm{N}$ que foi perdida do fertilizante mineral.

\section{$\mathrm{N}$ reciclado}

A aveia-preta cultivada em 2006, em todos os sistemas de culturas sem $\mathrm{N}$, apresentou diferentes quantidades de $\mathrm{N}$ acumulado em sua biomassa aérea, variando de 17 no Desc a $100 \mathrm{~kg} \mathrm{ha}^{-1}$ no G+M. As maiores quantidades de $\mathrm{N}$ acumulado pela aveia foram encontradas nos sistemas com maiores estoques de $\mathrm{N}$ total, demonstrando o efeito residual das leguminosas no aumento da disponibilidade de $\mathrm{N}$ às gramíneas (Figura 3). Esse efeito também foi demonstrado por Amado (1997), que observou que o histórico de 


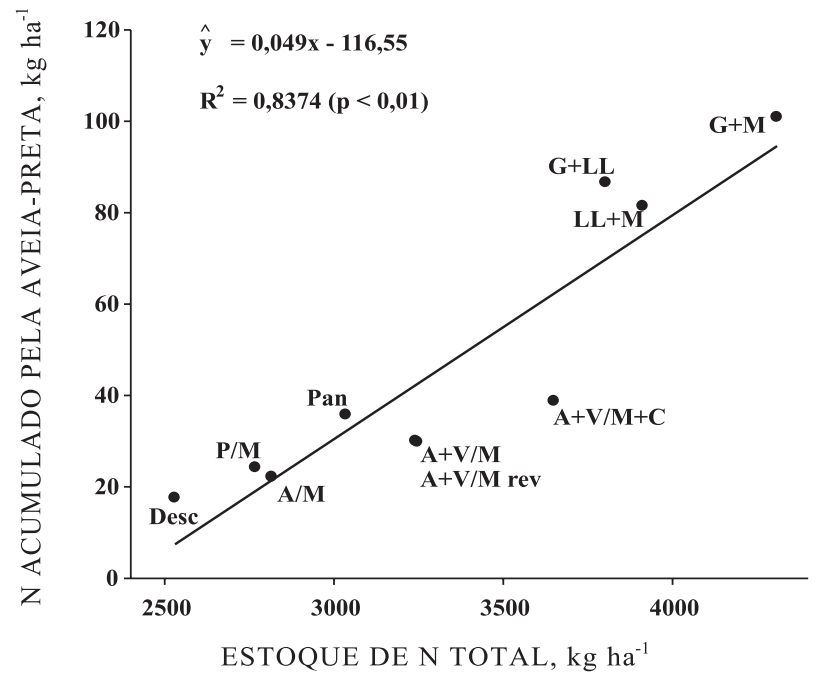

Figura 3. Quantidade de $\mathrm{N}$ acumulado pela parte aérea da aveia-preta, cultivada em 2006, em função dos estoques de $\mathrm{N}$ total na camada 0 $20 \mathrm{~cm}$, em 2005, em diferentes sistemas de culturas, com histórico de ausência de adubação nitrogenada mineral. A: aveia, $C$ : caupi, Desc: descoberto, G: guandu, LL: lablabe, M: milho, $P$ : pousio, Pan: pangola, rev: revolvido e V: ervilhaca.

nove anos de uso de leguminosas refletiu-se positivamente na absorção de $\mathrm{N}$ pelo milho cultivado em parcelas sem resíduos vegetais. O autor observou que o sistema aveia + ervilhaca/milho + caupi proporcionou um aumento de $11 \mathrm{~kg} \mathrm{ha}^{-1}$ de $\mathrm{N}$ absorvido pelo milho quando comparado ao sistema aveia/milho. Pal \& Shehu (2001) também observaram efeito positivo do cultivo de quatro anos de lablabe sobre o rendimento e a quantidade de $\mathrm{N}$ acumulado pelo milho em relação ao cultivo também de quatro anos de sorgo.

\section{CONCLUSÕES}

Na avaliação de 22 anos de uso de diferentes sistemas de culturas sobre o balanço de $\mathrm{N}$, além do rendimento de grãos de milho, nas condições edafoclimáticas de um Argissolo Vermelho distrófico típico da região da Depressão Central do RS, concluise que:

1. A utilização de leguminosas aumenta o rendimento de grãos de milho quando da ausência de adubação nitrogenada mineral.

2. A utilização de sistemas de culturas em plantio direto com leguminosas promove acúmulos de $\mathrm{N}$ no solo em relação a sistemas constituídos apenas por gramíneas.

3. A adubação nitrogenada promove aumento nos estoques de $\mathrm{N}$ total, porém com efeito menor do que em relação à adoção de leguminosas.
4. Maior estoque de $\mathrm{N}$ total determina mais quantidade de $\mathrm{N}$ acumulado pela aveia-preta.

5. O aumento da disponibilidade de $\mathrm{N}$, pela utilização de leguminosas ou pela aplicação de adubo nitrogenado mineral, promove aumento na quantidade de $\mathrm{N}$ exportado pela colheita de grãos de milho.

6. As leguminosas estivais apresentaram maior quantidade estimada de $\mathrm{N}$ fixado, que variou de $69 \mathrm{a}$ $90 \mathrm{~kg} \mathrm{ha}^{-1} \mathrm{ano}^{-1}$, do que as leguminosas de inverno, que apresentaram uma fixação média de $38 \mathrm{~kg} \mathrm{ha}^{-1}$ ano $^{-1}$ de $\mathrm{N}$.

7. Houve perda de $48 \%$ do $\mathrm{N}$ aplicado via fertilizante mineral na cultura do milho em 22 anos.

\section{LITERATURA CITADA}

AITA, C. Dinâmica do nitrogênio no solo durante a decomposição de plantas de cobertura: Efeito sobre a disponibilidade de nitrogênio para a cultura em sucessão. In: FRIES, M.R. \& DALMOLIN, R.S.D., coords. Atualização em recomendação de adubação e calagem - ênfase em plantio direto. Santa Maria, Palotti, 1997. p.76-111

AITA, C.; BASSO, C.J.; CERETTA, C.A.; GONÇALVES, C.N. \& DA ROS, C.O. Plantas de cobertura de solo como fonte de nitrogênio ao milho. R. Bras. Ci. Solo, 25:157-165, 2001.

ALVES, B.J.R.; ZOTARELLI, L.; ARAÚJO, E.S.; FERNANDES, F.M.; HECKLER, J.C.; MEDEIROS, A.F.A.; DODDEY, R.M. \& URQUIAGA, S. Balanço de $\mathrm{N}$ em rotação de culturas sob plantio direto em Dourados, MS. Seropédica, Embrapa Agrobiologia, 2005. 21p. (Boletim de Pesquisa e Desenvolvimento)

AMADO, T.J.C. Disponibilidade de nitrogênio para o milho em sistemas de cultura e preparo de solo. Porto Alegre, Universidade Federal do Rio Grande do Sul, 1997. 201p. (Tese de Doutorado)

AMADO, T.J.C.; BAYER, C.; ELTZ, F.L.F. \& BRUM, A.C.R. Potencial de culturas de cobertura em acumular carbono e nitrogênio no solo no plantio direto e a melhoria da qualidade ambiental. R. Bras. Ci. Solo, 25:189-197, 2001.

BAYER, C. Aumento do estoque de matéria orgânica de um Cambissolo Húmico catarinense sob plantio direto. R. Ci. Agroveter., 2:88-96, 2003.

BAYER, C. Dinâmica e qualidade da matéria orgânica em sistemas de manejo de solos. Porto Alegre, Universidade Federal do Rio Grande do Sul, 1996. 240p. (Tese de Doutorado)

BAYER, C. \& MIELNICZUK, J. Nitrogênio total de um solo submetido a deferentes métodos de preparo e sistemas de culturas. R. Bras. Ci. Solo, 21:235-239, 1997.

BAYER, C.; SPAGNOLLO, E.; WILDNER, L.P.; ERNANI, P.R. \& ALBUQUERQUE, J.A. Incremento de carbono e nitrogênio num Latossolo pelo uso de plantas estivais de cobertura de solo. Ci. Rural, 33:469-475, 2003.

BOHLOOL, B.B.; LADHA, J.K.; GARRITY, D.P. \& GEORGE, T. Biological nitrogen fixation for sustainable agriculture: A perspective. Plant Soil, 141:1-11, 1992. 
BURLE, M.L.; MIELNICZUK, J. \& FOCCHI, S. Effect of cropping systems on soil chemical characteristics, with emphasis on soil acidification. Plant Soil, 190:309-316, 1997.

CALEGARI, A.; MONDARDO, A.; BULISANI, E.A.; WILDNER, L.P.; COSTA, M.B.B.; ALCÂNTARA, P.B.; MIYASAKA, S. \& AMADO, T.J.C. Adubação verde no Brasil. 2.ed. Rio de Janeiro, AS-PTA, 1993. 346p.

CANTARELLA, E. Nitrogênio. In: NOVAIS, R.F.; ALVAREZ V., V.H.; BARROS, N.F.; FONTES, R.L.F.; CANTARUTTI, R.B. \& NEVES, J.C.L., eds. Fertilidade do solo. Viçosa, MG, Sociedade Brasileira de Ciência do Solo, 2007. p.375470 .

DA ROS, C.O. \& AITA, C. Espécies de inverno na cobertura de solo e fornecimento de nitrogênio ao milho em plantio direto. R. Bras. Ci. Solo, 20:135-140, 1996.

DIEKOW, J.; MIELNICZUK, J.; KNICKER, H.; BAYER, C.; DICK, D.P. \& KÖGEL-KNABNER, I. Soil C and N stocks as affected by cropping systems and nitrogen fertilization in a Southern Brazil Acrisol managed under no-tillage for 17 years. Soil Till. Res., 81:87-95, 2005.

ELLERT, B.H. \& BETTANY, J.R. Calculation of organic matter and nutrients storage in soils under contrasting management regimes. Can. J. Soil Sci., 75:529-538, 1995.

EMPRESA BRASILEIRA DE PESQUISA AGROPECUÁRIA EMBRAPA. Centro Nacional de Pesquisa de Solos. Sistema brasileiro de classificação de solos. Rio de Janeiro, 1999. $412 \mathrm{p}$

FERNANDES, S.B.V. Disponibilidade e eficiência de uso de nitrogênio pelo milho em sistemas de cultura. Porto Alegre, Universidade Federal do Rio Grande do Sul, 1998. 137p. (Tese de Doutorado)

FUGITA, K.; OFOSU-BUDU, K.G. \& OGATA, S. Biological nitrogen fixation in mixed legume-cereal cropping systems. Plant Soil, 141:155-175, 1992.

GAVA, G.J.C.; TRIVELIN, P.C.O.; OLIVEIRA, M.V.; HEINRICHS, R. \& SILVA, M.A. Balanço de nitrogênio da uréia $\left({ }^{15} \mathrm{~N}\right)$ no sistema solo-planta na implantação da semeadura direta na cultura do milho. Bragantia, 65:477486, 2006.

LEDGARD, S.F. \& STEELE, K.W. Biological nitrogen fixation in mixed legume-grass pastures. Plant Soil, 141:137-153, 1992.

LOVATO, T. Dinâmica do carbono e nitrogênio do solo afetada por preparos de solo, sistemas de cultura e adubo nitrogenado. Porto Alegre, Universidade Federal do Rio Grande do Sul, 2001.150p. (Tese de Doutorado)

LOVATO, T.; MIELNICZUK, J.; BAYER, C. \& VEZZANI, F. Adição de carbono e nitrogênio e sua relação com os estoques no solo e com o rendimento do milho em sistemas de manejo. R. Bras. Ci. Solo, 28:175-187, 2004.
MEDEIROS, J. C. Sistemas de culturas adaptados à produtividade, recuperação e conservação do solo. Porto Alegre, Universidade Federal do Rio Grande do Sul, 1985. 89p. (Dissertação de Mestrado)

MOREIRA, F.M.S. \& SIQUEIRA, J.O. Microbiologia e bioquímica do solo. 2.ed. Lavras, Universidade Federal de Lavras, 2006. 729p.

MORENO, J.A. Clima do Rio Grande do Sul. Porto Alegre, Secretaria da Agricultura. Diretoria de Terras e Colonização, Seção de Geografia, 1961. 46p.

PAL, U.R. \& SHEHU, Y. Direct and residual contributions of symbiotic nitrogen fixation by legumes to the yield and nitrogen uptake of mayse (Zea mays L.) in the Nigerian Savannah. J. Agron. Crop Sci., 187:53-58, 2001.

PEOPLES, M.B.; HERRIDGE, D.F. \& LADHA, J.K. Biological nitrogen fixation: An efficient source of nitrogen for sustainable agricultural production? Plant Soil, 174:3-28, 1995.

POUDEL, D.D.; HORWATH, W.R.; MITCHELL, J.P. \& TEMPLE, S.R. Impacts of cropping systems on soil nitrogen storage and loss. Agric. Systems, 68:253-268, 2001.

RAO, M.R. \& MATHUVA, M.N. Legumes for improving maize yields and income in semi-arid Kenya. Agric. Ecosyst. Environ., 78:123-137, 2000.

SAINJU, U.M.; SINGH, B.P. \& WHITEHEAD, W.F. Longterm effects of tillage, cover crops, and nitrogen fertilization on organic carbon and nitrogen concentrations in sandy loam soils in Georgia, USA. Soil Till. Res., 63:167-179, 2002 .

TEDESCO, M.J.; GIANELLO, C.; BISSANI, C.A.; BOHNEN, H. \& VOLKWEISS, S.J. Análises de solo, plantas e outros materiais. 2.ed. Porto Alegre, Universidade Federal do Rio Grande do Sul, 1995. 174p.

VIEIRA, F.C.B. Estoques e labilidade da matéria orgânica e acidificação de um Argissolo sob plantio direto afetados por sistemas de cultura e adubação nitrogenada. Porto Alegre, Universidade Federal do Rio Grande do Sul, 2007. 139p. (Tese de Doutorado)

WEBER, M.A. Leguminosas e os estoques de carbono e nitrogênio do solo em experimento de longa duração. Porto Alegre, Universidade Federal do Rio Grande do Sul, 2008. 122p. (Dissertação de Mestrado)

ZANATTA, J.A.; BAYER, C.; DIECKOW, J.; VIEIRA, F.C.B. \& MIELNICZUK, J. Soil organic carbon accumulation and carbon costs related to tillage, cropping systems and nitrogen fertilization in a subtropical Acrisol. Soil Till. Res., 94:510-519, 2007.

ZONTA, E.P. \& MACHADO, A.A. Sistema para análise estatística para microcomputadores - SANEST. Pelotas, Universidade Federal de Pelotas, 1984. 109p. 
